\title{
Fatigue life prediction of the SAE 1045 medium carbon steel using the acoustic emission technique associated with Weibull distribution approach
}

\begin{abstract}
This paper presents the capability of acoustic emission (AE) technique in predicting the fatigue life of the SAE 1045 carbon steel. Using the Weibull distribution approach, the specific AE parameters and the number of cycles to failure of the tested specimens were correlated. In addition, the lives of specimens were also calculated using the available empirical models. The AE and strain signals that were used were experimentally measured using the AE sensor and strain gauge, as the sensors were attached to the specimen during the fatigue test. The AE parameter was transform to the Wei bull parameter as this technique gives more accurate values. The results showed that the $\mathrm{AE}$ technique has a good potential in assessing the fatigue life with the designed h-N curve, correlating the AE hits-number and also cycles to failure.
\end{abstract}

Keyword: Fatigue life; Acoustic emission; Weibull distribution; Steel; Prediction 